underway, and formal publication of detailed results is expected later this year. Although the researchers studying this core have not found a mantle full of methane, they have found out much about the origin of this gas in the deep oceanic crust. Considering that serious uncertain- ties still exist about the origin of $\mathrm{CH}_{4}$ in natural gas reservoirs ${ }^{10}$, these findings are an important advance.

William C. Evans is at the US Geological Survey, Menlo Park, California 94025, USA.

\title{
Towards the ideal detector
}

\section{Francesco Paresce}

AstronOMERS are uniquely dependent on their ability to wring the most information possible from the usually small number of photons received from the cosmos. Ideally, what one needs is an accurate and reliable determination of the spatial location, the time of arrival, the energy and the polarization of each incoming photon.

In practice, this task is accomplished by a number of more or less complex devices that measure, with varying degrees of success, each of these parameters separately while ignoring the others. It has been a cherished dream of observational astronomers to be able to compress the entire measurement process down to a single element that could perform the operation in one fell swoop. A large step towards this goal has been taken with the achievement, reported on page 135 of this issue $^{1}$ by Peacock et al., of single-photon counting with simultaneous energy discrimination, using a niobium-based superconducting tunnel junction (STJ) at visible wavelengths. This development shows the way towards energy- and timesensitive imaging detectors, for astronomy and for other fields such as medical imaging and remote sensing where these characteristics may be useful.

Energy discrimination by itself is conventionally achieved by using filters to constrain the range of wavelengths reaching the detector, or, for higher spectral resolution, through the use of prisms or diffraction gratings to separate the different wavelengths spatially before detection. The introduction of many extra optical and mechanical elements before the actual detection, however, results in an unavoidable and considerable loss of sensitivity and accuracy.

Recently, imaging spectrometers have been developed that combine optomechanical image slicers and long-slit spectrometers to obtain a true threedimensional capability ${ }^{2}$ (the two dimensions of the observing plane plus the spectral dimension). They offer, at least in the infrared, considerable improvement in observing efficiency but are still far from ideal. Single-photon counting with imaging capability can be achieved with a number of devices ${ }^{3}$, but they are all extremely limited in efficiency and speed. All these methods suffer from the physical separation and inherent inefficiencies of the various measurement processes.

The use of the STJ should relieve, at least in principle, many if not most of these limitations. As an astronomical detector, it follows naturally from the introduction of semiconductor detectors such as charge-coupled devices (CCDs). In such silicon-based devices, the band gap between the ground state and the state excited by the absorption of a photon is comparable to the photon energy. As a consequence, only a single electron is extracted from the detector per absorbed photon, irrespective of the photon energy.

In contrast, the band gap of superconducting niobium as used in the new device is some three orders of magnitude lower, which means that of the order of 1,000 electrons are released per photon. More importantly, although the physics of the STJ detector differs in detail from that of semiconductors (and involves release of charge through the breaking of superconducting Cooper pairs), the average charge generated by an absorbed photon is proportional to its energy. By measuring the charge released, each detected photon can be sorted in energy, to an accuracy limited by the statistical fluctuations in the charge released. The overall quantum efficiency (the proportion of incoming photons detected) is limited only, in principle, by the transmission or reflection properties of the substrate. The STJ efficiency of $\sim 50$ per cent compares well with the best CCD devices and is many times higher than any ultraviolet-sensitive device available.

Although the wavelength resolution achieved by Peacock et al. $(\mathrm{d} \lambda \approx 45 \mathrm{~nm}$ at $350 \mathrm{~nm}$ ) is modest by conventional spectroscopic standards, this is only the beginning of the development of the STJ as an astronomical detector. Other materials have even lower band gaps than niobium, and therefore promise to yield STJ devices with much higher spectral resolution. Peacock and co-workers are actively pursuing these materials and have already improved on their niobium results with a tantalum-based device. It is unlikely, however, that high spectral resolutions can be achieved soon, so spectroscopy with $\mathrm{d} \lambda / \lambda$ $\geqslant 1,000$ will still have to be performed using conventional spectrographs. STJs could be envisaged as extremely effective spectral order sorters or used in conjunction with Fabry-Perot-type spectrographs. Their ideal domain of application for now and the foreseeable future is in extremely sensitive continuum observations with moderate resolution.

As one might expect, however, this ability to discriminate photons in energy and arrival time without the use of filters or dispersive elements comes at a price. In order to distinguish the relatively feeble charges generated by the STJ detector above thermal background noise, such devices must operate at extremely low temperatures, typically below $1 \mathrm{~K}$. This requires highly sophisticated cryogenic technology. Moreover, each pixel needs its own amplifier kept at the same temperature, so arrays of many pixels quickly become a topological nightmare to make without producing crosstalk. The device is also very sensitive to acoustic noise and vibrations and to magnetic contamination. Finally, there are count-rate limitations aggravated by sensitivity to low-energy infrared photons, which may demand some kind of filtering that reduces the overall sensitivity of the device. None of these problems is insurmountable, but they will all need very careful attention in order to reduce or eliminate their effects.

Among the many different applications that can be envisaged for this detector, a high priority ${ }^{4}$ is its use in space, in conjunction with a sophisticated ultraviolet to infrared-sensitive telescope with very high spatial resolution and high sensitivity owing to its low background. In particular, it would enable the Hubble Space Telescope to perform spectral-energy-distribution and redshift measurements of the galaxies it can now only detect by broadband imaging. Such an HST-based STJ camera, of perhaps 100 by 100 pixels, may be the only hope on the horizon of accurately measuring the redshifts of most of the 3,000-plus galaxies with visual magnitudes of 27 to 28 that were detected in the Hubble Deep Field.

Even on the ground, these devices will be able, for example, to image a stellar cluster with an 8-m-diameter telescope and in a few minutes obtain not just the conventional colours and magnitudes of all the stars in the field but also their continuum spectra with $10 \AA$ resolution. This promises a true revolution in the way astronomers work in the future.

Francesco Paresce is at the European Southern Observatory, Karl Schwarzschild Strasse 2, Garching bei München, 85748, Germany.

\footnotetext{
1. Peacock, A. et al. Nature 381, 135-137 (1996).

. Weitzel, L. et al. Astr. Astrophys. (in the press)

3. Joseph, C. L. Expl Astr. 6, 97-127 (1995).

4. Newsletter No. 23 (Space Telescope/European

Coordinating Facility, Garching, Sept. 1995).
} 